\title{
On Smart-Care Services: Studies of Visually Impaired Users in Living Contexts
}

\author{
Ying Liu, Jean Bacon \\ Computer Laboratory \\ University of Cambridge \\ JJ Thomson Avenue, Cambridge CB3 0FD, UK \\ firstname.lastname@cl.cam.ac.uk
}

\author{
Roger Wilson-Hinds \\ Choice Technology (UK) Limited \\ 7 The Rookery, Orton Wistow \\ Peterborough, PE2 E6YT, UK \\ roger@ screenreader.co.uk
}

\begin{abstract}
Smart care technology is any sensor based technology used to aid and support human independent living. Such technologies offer new potential and can give rise to new problems for making the technology accessible to users. In this work we focus on integrated services for people with visual impairment. Web based information services have already been adapted for people with varying degrees of disability. What is needed now is a service oriented architecture that integrates information services with smart care technology such as sensor devices that generate data for input, processing, storage and query. The main new challenge we identified here is that users may be living in a perplexing contexture - a chain of barriers affecting their ability to live independently. Contexts such as mobility dependencies must therefore be recorded and used, indoors and outside. Users should be monitored during their interactions with services, and the meaning of their behaviour inferred in order to refine the services. Moreover, by giving a set of user scenarios, we present a higher level view of users' needs than single service invocation; alternatives and follow-on services might be suggested and previous interactions built upon. Management of the architecture must allow for incorporation of new technology and upgrade of services. Technology for smart care is developing rapidly; its usefulness, and acceptance, requires a dynamic and flexible architecture to support ease of management and use.
\end{abstract}

Smart Care; Smart Service; Sensor Applications; Independent Living; Living Contexts; Visually Impaired; Universal Access; Inclusive Design; HCI.

\section{INTRODUCTION}

People in general are becoming increasingly mobile, not only moving or travelling in physical space from one location to another more often than ever before, but also using virtual space to undertake a task seamlessly without regard to geographical boundaries, e.g. online booking, shopping and banking. But this capability may be severely limited for those ("users" hereafter) who have lost sight, have severe dyslexia or visual limitations that contribute to confusion in orientation when travelling, or elderly people who have visual pathologies.

Universal access in a digital society is about enabling individuals, with varying degrees of less than perfect ability, to access information or facilities they require in order to function, as they choose, in their environments. It also identifies any barriers to this access, which can arise from the technologies themselves or from exclusive social contexts [9][15]. There are proactive approaches based on various metaphors [1], as well as useful techniques and methods, e.g. using ontology based semantic annotation to semiautomatically identify objects and discover their roles in support of mobility [21].

Smart care technology is sensor based technology used to aid and support human independent living [23]. Such technologies offer new potential and can give rise to new problems for universal access. Rapid deployment of wireless sensor technologies ${ }^{1}$, together with hand-held and ubiquitous computing[16], sensor networks[20], data communication networks [18], mobile ${ }^{2}$ and wearable smart devices [7], make it possible to provide services for smart homes or remote healthcare monitoring [19]. These technologies will have a profound impact on the type, content, location, operation and functionality of care products and services [7], and all this must be integrated with the earlier information services. It is therefore crucial to adopt the concept of universal access at this initial stage of smart care research.

\footnotetext{
${ }^{1}$ http://www.antd.nist.gov/wctg/manet/adhoclinks.html

2 http://www.about-smart-phones.com/
} 
To develop smart care for users, we argue that an innovative approach is required so that the increasingly specialised devices can be supported by a flexible architecture for dynamic, non-text reading services.

A service is provisioned in order to give value to a client, for example, to book a train ticket for a given date. This service is the provision of a train ticket (entity) that is to be used according to the specified constraints. A web-based service [6][8][11] is a computational entity, in this case for accessing a timetable, selecting and receiving a train ticket and making a payment over the Internet. The Service Oriented Computing paradigm [2] has been widely adopted but it does not yet fully address largescale system design [3]. Also, end-user services must be easy to use, and personalised or context-aware while in use [1] and, most importantly, must be secure and trustworthy [4][5]. Smart care services must have all of these features.

But unlike current web based services which are mainly developed for reading textual information (e.g. train timetables, ticket costs), smart care services require an increasing number of sensory devices with greater specialisations. They are driven by context - where the devices are used and how the data are acquired, processed and distributed. Thus, the specialisation of a device must be able to address the wider question of the provision of the service required to support its function. For example, Portable Braille PDAs are already available as plug-ins for PCs and GPS ${ }^{3}$ (Global Positioning Systems), but are not yet widely used. This is, most likely, due to the lack of sufficient services to support these devices. Such a service oriented approach must be integrated with an adequate architecture. We shall discuss this further in the following three sections.

In section 2, we address related work and our research motivations. In section 3, we illustrate user scenarios where the desired interactions between users and a smart environment are complex. Assistance should be offered appropriate to users' behaviour and context; alternatives might be suggested. As we expand below, someone with poor health may not go to see a doctor regularly, because of the difficulty of using a bus or of finding their way around a hospital. Home based smart care services may offer an alternative. We conclude these findings in section 4.

\section{INTUITIVE AND CONTINUITY PROBLEMS OF MOBILITY}

The Guide Dogs organisation conducted focus groups and interviews across the UK between May and September 2005, investigating users' perspectives on the provision of audiovisual information about buses [10]. The participants of the focus groups included people with

${ }^{3}$ http://www.pulsedata.com/Products/Notetakers/braillenotepk.asp visual, hearing and dual sensory impairments, and those over 65 . There was unanimous agreement that the ability to identify the right bus at the outset of one's journey, including sufficient information to enable adequate pre-journey preparation, is fundamental to a stress-free and successful journey. Several participants spoke of having 'great difficulty identifying what bus to get on' or of 'not being able to see the buses in the distance' or of 'terrible problems consistently missing buses, most especially when buses are lined up one behind the other and it becomes quite impossible to identify which is which. Being able to locate the wanted bus easily and independently, even among a row of several buses, clearly emerged as a priority for these users.

There are many relevant technologies that potentially can be used as mobility aids, e.g.:

- Sonar Canes ${ }^{4}$, Sonic Guide and Sonic Pathfinder ${ }^{5}$ are products for detecting obstacle and hazards.

- vOICe $^{6}$ developed a range of devices that translate arbitrary video images from a regular PC camera into sounds in the hope that users can "see with their ears". Such a device scans each camera snapshot from left to right, while associating height with pitch and brightness with loudness.

- Talking $\operatorname{Sign}^{7}$ is an electronic aid used to assist in orientation and navigation. The system uses infrared signals from permanently installed transmitters, to a handheld receiver, that decodes the signal and delivers a voice message through its speaker or headset. Transmitters can be placed on signs, buildings, doors etc.

- GPS-talks, BrailleNote GPS MoBIC ${ }^{8}$, and Visuaide Treker ${ }^{9}$ are navigation systems that utilise GPS and wireless communication networks for outdoor wayfinding.

- Cricket $^{10}$ is an indoor location system for pervasive and sensor-based computing environments providing finegrained location information - space identifiers, position coordinates, and orientation, to applications running on handhelds, laptops, and sensor nodes. Cricket is intended for use indoors or in urban areas where outdoor systems like GPS don't work well. Reportedly, it can provide distance ranging and positioning precision of between 1 and $3 \mathrm{~cm}$.

The Ministry of Economy, Trade and Industry (METI) of Japan started a project for developing user friendly wayfinding/guidance systems for various disabilities ${ }^{11}$. The main objective is to develop a single device which can be used

\footnotetext{
${ }^{4} \mathrm{http} / / /$ www.batforblind.co.nz/history.htm

5 http://www.vard.org/jour/99/36/4/lagrow.htm

${ }^{6} \mathrm{http}: / /$ www.seeingwithsound.com/voice.htm

7 http://talkingsign.co.uk/

$8 \mathrm{http}: / /$ www.SenderoGroup.com/

9 http://www.visuaide.com/

${ }^{10} \mathrm{http}: / /$ cricket.csail.mit.edu/

${ }^{11} \mathrm{http}: / /$ www.csun.edu/cod/conf/2005/proceedings/2210.htm
} 
for different types of wayfinding/guidance systems. The common platform architecture to be designed integrates functions that operate with different types of sensors and media such as GPS, Infrared Communication, Radio Communication and RFID. The platform can also incorporate new sensors' functionalities. The project is to propose the standardized system to ISO.

However, these devices and systems tend to be designed to help users to become familiar with an outdoor environment. They are not intuitive enough for new or unknown places or for access to an unfamiliar social infrastructure such as a hospital or shopping mall. They may be based on users hearing sounds bouncing off nearby objects (ultrasonic echolocation), creating a familiar pattern as the person moves, but offering little or no information regarding the objects themselves.

Users encounter objects in order to travel. That is, they must not only avoid obstacles, but also be able to find objects, such as buses, buildings and entrances. It is unclear whether such object awareness is the focus of other research programs. For example, the ASK-IT ${ }^{12}$ integrated project aims at establishing Ambient Intelligence in semantic web enabled services, "to support and promote the mobility of mobility-impaired people, enabling the provision of personalised, self-configurable, intuitive and context-related applications and services and facilitating knowledge and content organisation and processing".

Researchers have demonstrated that information about an object and its location can be described and encoded in a RFID tag associated with the object. Users can receive information about the object through wearable devices that embed RFID readers [21]. Reportedly, a ubiquitous computing network has been developed to assist users in finding their way around indoor spaces [22]. It is based on the "Cyber Crumb" concept: the idea that tiny, inexpensive solar-powered digital chips can be used to store relevant pieces of information that can be placed along building walkways, like a trail of crumbs to follow. A wireless network of "crumbs" provides access from any point in a building to a central server that provides orientation and wayfinding information.

A location-aware sensing approach can also be used in developing applications for tracking objects, This has already been exploited in industry for resource discovery, human or robot navigation, computer games, medical applications (e.g., equipment and patient tracking or monitoring), or migration of a stream of objects.

In summary, these enabling, object-aware technologies are limited to helping users to interact with events in the environment, and are thus unable to provide specific navigation assistance in real time. It is important to remember that users' mobility is often goal driven.

\footnotetext{
${ }^{12} \mathrm{http}: / /$ www.ask-it.org/
}

Dynamically correlating much of the interactive information between users, objects and the environment is a critical prerequisite for assisting individuals' travels.

Therefore a more innovative approach is urgently required to integrate existing technology as "vision aids and vision substitution" towards a multi-dimensional, service-oriented approach. The aim is real time, personalised, non-textual reading, integrated sensory information and communication services, to augment sensory perceptions about objects, indoors and outside. There are other important reasons for this device and service integration.

Firstly, although it is necessary to know the various devices' special properties, there are too many alternative use cases to make bespoke solutions feasible. A service platform is needed to allow devices to "plug-and-play" to meet the specific needs of the various users, e.g., a device which suits a partially vision-impaired person can be completely useless for a totally blind person. Many contexts may be more difficult to understand, and can be inadequately represented in a phase of device specialisation. Consider the following combinations of many users and many disadvantageous situations (reported in [10]),

"About 24,000 people in the UK are registered as deafblind $^{13}$. When devices add new features, the relevant services may have to adopt the changes, e.g. incorporating vibration-tones and downloadable information in Braille formats. Approximately 10 million people in the UK have a disability. ${ }^{14}$ Of this population, up to $70 \%$ have difficulty walking or climbing steps, an estimated $41 \%$ have a hearing loss, and $24 \%$ a vision loss. ${ }^{15}$ A combined total of approximately three million people in the UK have sight loss, dyslexia or other reading-related disability. ${ }^{16}$ The strong correlation between disability and age (up to onethird of the population aged between 50 and 60 have a disability) also means that the prevalence of disability in society is set to grow with predicted demographic changes in the population."

Secondly, with respect to the above combinations of users, there are many inter-related environmental contexts; the need should not be considered in isolation. For example, a blind person may need to use a bus in order to see a doctor, or to visit their bank manager; access to a bus is a need. From a wider perspective, a service gateway might suggest home based healthcare or online banking services, as well as providing an information service on bus travel.

Thirdly, the management of emerging technologies must be sustainable and coordinated, and this cannot depend only on applied research dedicated to the preservation of the technologies or, more significantly, on an organisation dedicated to changing services as technologies improve. A

\footnotetext{
${ }^{13} \mathrm{http}: / /$ www.deafblind.org.uk/deafblindness/index.html

${ }^{14} \mathrm{http}: / / \mathrm{www} . \mathrm{drcgb}$. org/thelaw/ casedetails.asp?category=legal\&id=521

${ }^{15}$ Ibid.

${ }^{16} \mathrm{http}: / /$ www.rnib.org.uk/xpedio/groups/public/ documents/publicwebsite/public_r2rreport.hesp
} 
service architecture must be able to incorporate a new technology, upgrade a service, or create a new one. Many lessons can be learned from the past. For example, reportedly ${ }^{17}$, as computer technology was becoming cheaply available and effectively deployable, the Nottingham Navigation Centre, a world-leading institution that focused on blind navigational technologies, was closed for lack of funding. If a service architecture had been in place, components of many ground breaking technologies that came out of the research might have been re-used, such as the sonic Pathfinder.

\section{DYNAMIC SERVICE SCENARIOS}

As we have discussed, mobility-needs may exist in a perplexing contexture. What is needed is an integrated service, scalable to applications of technologies. We summarise the user living contexture as follows.

Sight needs are for vision enhancement and substitution. Solutions are sought through medical, cognitive, or other means to enable users to identify an object or to navigate in a built environment.

Daily living needs are for the maintenance or improvement of the quality of life. Since over half of visually impaired persons live alone, they have to cope with personal care, cooking, shopping, using ticket selling machines or cash dispensers.

Critical needs are obviously for health care, emergency aids or safety at home.

Individual needs are needs based on choice and the pursuit of one's wishes, interests, preferences, values and aspirations in different spheres and phases of life, e.g., driving a car, playing a game, music or sport.

It should be noted that daily living and critical requirements must be met whether or not a person has a disability. Also, at the scale of a single application, it is essential to study the practical implications of users' problems in detail. At the scale of several applications, users' needs must be distinguished and categorised so that we can distribute the technologies in users' living contextures.

We propose designs for the following four user scenarios, showing that desired interactions between users and a smart environment require an architecture of dynamic and heterogeneous components.

\section{A. A Talking Gadget}

Helen is 63 and lives alone. She is totally blind and has diabetic retinopathy (a side effect of diabetes that is a leading cause of blindness among the working-age population). Because of her poor health, she needs to see her doctor regularly. Helen has heard about CareGrid

\footnotetext{
${ }^{17} \mathrm{http} / /$ www.wayfinding.net/navigation.htm
}

services (Figure 1). She is cautious and sceptical about the services and having the computer and wireless kit in her home. Her other worry is how to find them if she drops them somewhere or she forgets where she has put them. However the program manager arrives at her home and explains not only how easy it is to manage and use the equipment, but also that she can subscribe to other services to make her life easier. In particular, the device Talking Gadget with tone-and-shaped touch buttons recognises Helen's face and finger print, so nobody else can unlock and use it. The gadget does many other things. She can either rent or buy the hardware, and subscribe to or cancel the services at any time. So she joins the programme and subscribes to three services.

\section{B. Talking@Travel}

If she presses the round button, the gadget is in the travelFinder mode. Before travelling, she tells the gadget the destination name. If she is a first time traveller to this destination, the gadget makes a plan. If she is returning to a place she has already visited, the gadget updates the plan with real time information, and tells Helen how to get there and when she will arrive. Assuming a sensor-rich external environment, when she is waiting for a bus, the gadget identifies the right bus and announces the stops when Helen is on it. As she walks around the city it tells her about the buildings she is passing.

\section{Talking@Object}

If she presses the square button, the gadget is in the objectFinder mode. The gadget comes with an ample supply of thin electronic tags that it can read. Let us assume that the technology is able to detect a tag within house-wide range and that a database of room-names to 3D locations has been created. Helen can attach a tag to any object. She only needs to use the gadget to scan the tagged object once, then tells the gadget her preferred name for the object.

When she wants to know where an object is, she can ask the gadget, using this name. An alternative approach, perhaps more realistic for current technology, is that she walks around the house holding the gadget and is alerted when she comes close to the lost object. Helen can use the gadget as a "mirror" too. The gadget reads her tagged clothes and tells her their colours. She can match her new coat with a nice pair of trousers.

\section{Talking@Care}

If she presses the triangular button, the gadget is in the mode of careFinder. She can attach a set of medical devices supplied to get her health conditions checked: blood pressure, weight, pulse and blood glucose levels. The gadget wirelessly collects and transports the data to her home computer to be analysed; it also reminds her regularly to check a number of times a week at specific times. If she is not at home, the gadget stores the data temporarily and remotely transmits these to her surgery. She has asked the gadget to remember the devices in case she cannot find them. 


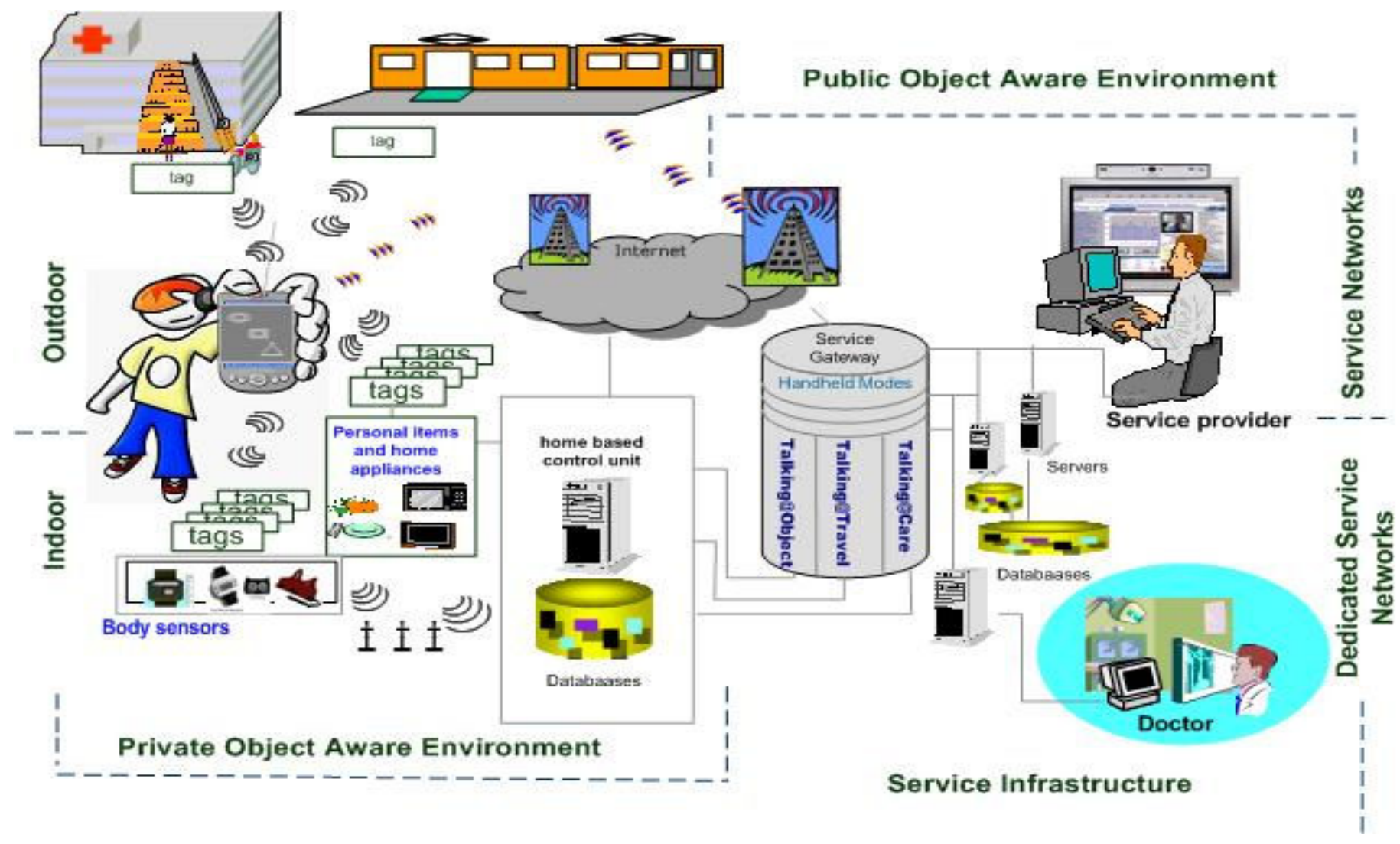

Figure 1. A talking gadget and services

When she goes out she uses the gadget to augment her guide dog. The gadget helps keep her informed of her changing environment, giving her greater control than ever before. The dog can help her avoid obstacles safely and can guide her home when commanded. Before subscribing to CareGrid, she always asked somebody to accompany her to hospital. Now she can make this trip more quickly and independently.

\section{E. Multi-functional gadget}

There is an incentive to invent a voice-commanded, object-aware mobile gadget with services, sufficiently and effectively connecting users via remote servers to mobility related objects in both physical and virtual space. The gadget has an embedded voice recognizer and sensory memory, infrared and RFID sensing, a data acquisition hub, and local data storage. It is capable of peer to peer networking, hosting third party sensors, sensing around personal surroundings indoors and outside, processing real time data and distributed data streaming. It can act like a personal server and a wireless data gateway programmed via a remote server to control and manage sensory data and objects. It has biometric security: face and fingerprint recognition. Users give commands to the gadget by voice, and can train the gadget to remember a tagged object by telling the name to the gadget. The remote server can manage users' contextual information, correlate public travel information and public infrastructural information, and offer Talking@Gadget services to users as mobility aids.

\section{CONCLUSIONS}

Visually impaired people currently still depend on very primitive aids such as canes, guide dogs or spoken directions for access to only known routes and social infrastructures. They have great difficulty in managing their daily lives and participating in social and health-critical activities. Current technologies may not be intuitive enough to be used for real world applications. Wireless sensor technologies and wearable mobile devices are rapidly integrating with ICT in providing real time information about the environment where people live. However, these advances are still largely unexplored for integrated smart care for blind and sight-impaired users. A more innovative approach is required to create accessible environments and new living spaces. The design scenarios show the potential of smart care technologies to improve blind and sightimpaired people's mobility, seamlessly, indoors and outside. 
To date, there is only a limited convergence towards a common user interface standard for mobile service appliances. The services we envisage require a new architecture along the following dimensions:

- thin clients: no client responsibility for software;

- a flexible, plug-and-play architecture;

- dependability, e.g. detection of easily misplaced and stolen items, biometric access control;

- mobile appliances that can store and access data;

- tactile input and output devices;

- converging functionality from many existing platforms.

\section{ACKNOWLEDGEMENT}

We gratefully acknowledge the support of EPSRC through the CareGrid grant EP/C53718.

\section{REFERENCES}

[1] P. L. Emiliani and C. Stephanidis, "Universal access to ambient intelligence environments: opportunities and challenges for people with disabilities," IBM Systems Journal, vol. 44, no. 3, 2003, pp.605-619.

[2] M. N. Huhns and M. P. Singh, "Service oriented computing: key concepts and principles." IEEE Internet Computing, vol. 9, Issue 1, Jan-Feb 2005, pp.75-81.

[3] J. Bacon, "Expectations and reality in distributed systems," Proceedings of IASTED International Conference on Parallel and Distributed Computing and Networks, Insbruck, Austria, February 2005, pp.vii xiv.

[4] J. Bacon and K. Moody, "Adaptive middleware: toward open, secure, widely distributed services," Communications of the ACM, vol.45, no.6, June 2002, pp.59-63.

[5] J. Bacon, K. Moody and W. Yao, "A model of OASIS role-based access control and its support for active security," ACM Transactions on Information and System Security (TISSEC), vol.5, no.4, November 2002, pp.492540.

[6] E. Cerami, Web Services Essentials. O'Reilly, 2002.

[7] ESTO: "Science and technology roadmapping: ambient intelligence in everyday life (AmI@Life)," JRC/IPT European Science and Technology Observatory Study. June 2003.

[8] D. Fensel, U. Keller, H. Lausen, A. Polleres and I. Toma, "WWW Or what's wrong with web service discovery," Proceedings of workshop on Frameworks in Web service, Innsbruck, Austria, June 2005.

[9] C. Maxwell, Global Trends That Will Impact Universal Access to Information Resources, UNESCO 15 July 2000.
[10] F. Nzegwu, "Audio and visual information on buses: moving toward Inclusion," Guide Dogs for the Blind Association, Reading, UK 2005.

[11] C. Preist, "A conceptual architecture for semantic web services," Proceedings of the International Semantic Web Conference (ISWC 2004).

[12] Queue, Building Web Services, ACM Queue March 2003.

[13] B. R. Schatz and J. B. Hardin, "NCSA Mosaic and the World Wide Web: global hypermedia protocols for the Internet," Science Magazine, no. 265, 12 August 1994, pp. 895-901.

[14] L. S. Star, "Got Infrastructure? how standards, categories and other aspects of infrastructure influence communication," Proceedings of The 2nd Social Study of IT Workshop at the LSE ICT and Globalization, London, 22-23 April 2002.

[15] C. Stephanids, "User interfaces for all: new perspectives into HCI," In: User Interfaces for All - Concepts, Methods and Tools, C. Stephanids, Eds, Lawerence Associates, Mahwah, NJ, 2001.

[16] G. D. Abowd, G. R. Hayes, G. Iachello, J. A. Kientz, S. N. Patel, M. M. Stevens and K. N. Truong, "Prototypes and paratypes: Designing mobile and ubiquitous computing applications," IEEE Pervasive Computing, Oct-Dec 2005, pp. 67-73.

[17] Zhao, F. \& Guibas, L. Wireless sensor networks. Elsevier Morgan Kaufmann Publishers, 2004.

[18] G. L. Bodic, Mobile Messaging, Technologies and Services, John Wiley \& Sons, Ltd., 2d. ed. 2005.

[19] G. Dewsbury, I. Sommerville, M. Rouncefield, K. Clarke, "Bringing IT into the home - a landscape documentary of assistive technology, smart home, telecare, telemedicine in the home in relation to dependability and ubiquitous computing," Computing Department, Lancaster University, UK 2002.

[20] B. Rhodes and K. Mase, "Wearable in 2005," IEEE Pervasive Computing, vol. 5, no.1 Jan-Mar 2006, pp. 9295.

[21] D. A. Ross, "Cyber crumbs for successful aging with vision loss," IEEE Pervasive Computing, vol.3, no.2. Apr-Jun 2004, pp. 30-35.

[22] Y. Yesilada, S. Harper, C. Goble and R. Stevens, "DANTE: Annotation and transformation of web pages for visually impaired users," WWW2004 ACM, May 2004. pp. 490-491.

[23] S. Giroux and H. Pigot, From Smart Homes to Smart Care: ICOST 2005 (Assistive Technology \& Research), IOS Press, USA, 2005. 